\title{
Author Index to Volume 34
}

\author{
Aalto TK, 572 \\ Ábrahám CS, 182 \\ Abrams SA, 455 \\ Adachi N, 258 \\ Ahuja S, 809 \\ Akil $\mathrm{H}, 646$ \\ Akselrod S, 134 \\ Albini $\mathrm{CH}, 435$ \\ Alex S, 288 \\ Alsat E, 439 \\ Andersson U, 213 \\ Andrews WL, 293 \\ Angenent FWM, 490 \\ Ank BJ, 544 \\ Anttilla R, 297 \\ Aperia A, 308 \\ Aprigliano M, 606 \\ Aquila WJ, 187 \\ Ascuitto RJ, 323 \\ Aubourg P, 270 \\ Aziz SM, 32 \\ Baan J, 178 \\ Baarsma R, 443 \\ Bada HS, 366 \\ Bảgenholm R, 23 \\ Baker L, 265 \\ Ban K, 105 \\ Baraldi E, 329 \\ Barbieri P, 329 \\ Barks JD, 192 \\ Barness LA, 552 \\ Bartelds B, 51 \\ Beauchamp GK, 805 \\ Beekhuis $\mathrm{H}, 154$ \\ Beitins IZ, 829 \\ Belai A, 354 \\ Benjamin DR, 341 \\ Bennett MJ, 89 \\ Benson LN, 38 \\ Ben-Yitzhak O, 79 \\ Berant M, 79 \\ Berger R, 443 \\ Bergman D, 312 \\ Berkenbosch A, 485 \\ Bernardini IM, 115 \\ Berry GT, 89 \\ Billings K, 611 \\ Birkenmeier EH, 837 \\ Biro FM, 555 \\ Blanco LN, 334 \\ Bock I, 460 \\ Bogaert GA, 755 \\ Bolton DPG, 518 \\ Bongers-Schokking JJ, 73 \\ Bonnin P, 796 \\ Bootsma AH, 277 \\ Borgers M, 379 \\ Bors Z, 742 \\ Bortolussi R, 243 \\ Bowron A, 10 \\ Boyd RDH, 661 \\ Braverman LE, 288 \\ Breidbart S, 300 \\ Breier BH, 62 \\ Bresser BW, 173 \\ Broekhuizen MLA, 44 \\ Brown MB, 829 \\ Bryson YJ, 66 \\ Buescher ES, 208 \\ Buhrfiend CM, 389
}

Burger R, 199

Burk RD, 300

Busija DW, 366

Butterworth RF, 777

Byrne GC, 785

Cairo MS, 56, 560

Caplan M, 222

Carlsson B, 217

Carrasco H, 670

Carver TD, 654

Carvey PM, 389

Chang S, 781

Chang YT, 698

Chanoine J-P, 288

Charnas LR, 115

Charpentier C, 129

Cheng S-N, 720

Chen Q-Y, 785

Chen TP, 544

Chen Y, 27, 577

Christova E, 624

Church MK, 159

Coalson JJ, 729

Coates PM, 111

Colier W, 15

Colon EJ, 73

Contrino J, 249

Cope M, 354

Corbett RJT, 370

Cotton RB, 495

Coy DH, 323

Cruz JR, 217

Cruz MR, 312

Curstedt T, 490

Curzi-Dascalova L, 624

Cutilli JR, 265

Dai Y, 159

Dalakas MC, 115

Dalinghaus M, 471

d'Allest AM, 624

Davis TH, 167

Dawson CA, 349

Dean TP, 159

DeGoede J, 485

de Graeff-Meeder ER, 424

de Groot CJ, 73

de Haan $\mathrm{HH}, 379$

de Haan J, 379

Dehan M, 624

DeJonge J, 781

deLemos DM, 729

deLemos RA, 729

Delpech B, 716

Delpy DT, 354

De Vivo DC, 28

Devlieger H, 642

de Zegher F, 642

Diamandi E, 675

Diamond E, 79

DiMauro S, 281

Dollberg S, 512

Donnai P, 661

Donnelly MM, 512

Douglas SD, 265

D'Souza SW, 66

During MJ, 187

Edwards AD, 354

Eggermont E, 642

Eiselt M, 624

Eisenman D, 634
Elliott SJ, 360

Enhorning G, 834

Eriksson UJ, 791

Esterman AL, 312

Evain-Brion D, 439

Evans PC, 62

Evans RA, 303

Eyskens FJ, 762

Fang SL, 288

Felder R, 124

Fessard C, 716

Feste AS, 318

Fiehn W, 772

Figueroa JM, 624

Filippone M, 329

Finckh B, 129

Finlay TH, 312

Fiorini R, 725

Fisher DA, 393

Forsum E, 448

Fouron J-C, 796

Foy T, 318

Frankenne F, 439

Frank L, 27, 334, 577

Freeman K, 318

Frey U, 478

Friederichs E, 688

Friedrich JB, 772

Friel JK, 293

Fryckstedt J, 308

Furukawa M, 237

Gahl WA, 115

Galland BC, 518

Garcia D, 370

Gaudier F, 303

Gaultier C, 624

Geffner ME, 66

Georgieff M, 565

Gerding AM, 471

Gibson KM, 129, 277

Gillespie MN, 32

Gillman MW, 555

Giorgi PL, 725

Girard N, 716

Gittenberger-de Groot AC, 44

Glazier JD, 661

Gluckman PD, 18, 62

Glueck CJ, 670

Goji K, 229

González-Cossio T, 217

Gospe Jr SM, 6

Gouyon J-B, 120

Graham BS, 167

Granger JP, 750

Gratama JWC, 471

Grogaard JB, 813, 821

Grohs B, 173

Groneck P, 586

Grubb J, 837

Gruber WC, 167

Gudnason V, 403

Guignard J-P, 120

Guillery EN, 124

Guimarâes H, 624

Gunn AJ, 18

Gurbindo C, 162

Haddad GG, 634

Hale DE, 89, 694

Hallberg L, 680

Halperin S, 243
Hamer T, 670

Hamori CJ, 1

Hansen TN, 360

Hanson LÄ, 217

Hanson WL, 596

Han VKM, 148

Harding JE, 62

Harding R, 148, 611

Harper RK, 618

Harper RM, 618

Hasaart THM, 379

Hashimoto T, 111

Haun SE, 530

Haworth SG, 591

Hay Jr WW, 654

Heales SJR, 10

Heidenreich RA, 416

Heikamp A, 154

Helfaer MA, 530

Hennen G, 439

Herías MV, 217

Higgins A, 837

Hirsch M, 134

Hoffmann GF, 129, 277

Hoogland RA, 73

Hooper SB, 148, 611

Horiuchi I, 385

Horiuchi S, 258

Houston R, 15

Howells DW, 10

Howlett S, 243

Hsueh W, 222

Hübner C, 129

Hultén L, 680

Humphries SE, 403

Hunninghake GW, 502

Hyland K, 10

Ichiki T, 105

llowite NT, 204

Indo $\mathrm{Y}, 111$

Iobidze M, 781

Israele $\mathrm{V}, 66$

Itoi T, 735

Iwamoto HS, 47

Iwamoto K, 698

Jacobson JD, 341

Jakobs C, 277

Jakubowska AE, 611

Jarvis JN, 781

Jenkins HR, 666

Johns DP, 611

Johnston JM, 237

Jones M, 606

Jones TW, 785

Jonzon A, 601

Jorch G, 173

Jose PA, 124

Jyonouchi H, 565

Kabir N, 448

Kadner SS, 312

Kadowitz PJ, 323

Kalhan SC, 80

Kantar A, 725

Kanyicska B, 742

Kappy MS, 698

Karin J, 134

Kasa-Vubu JZ, 829

Kelly DP, 694

Kilpatrick L, 265

Kirk KA, 303 
Kirsch JR, 530

Kivivuori SM, 297

Kjellmer I, 23

Klautz RJM, 178

Kletter GB, 829

Kliegman RM, 701

Knoppel E, 560

Knowles WJ, 785

Kobayashi M, 105

Koehler RC, 530

Koers JH, 471

Kogan BA, 755

Kohl B, 772

Kohlschütter A, 129

Kok RM, 277

Kolobow T, 606

Kondo N, 499

Korchak HM, 265

Korones SB, 366

Kovács J, 182

Kraemer R, 478

Krasnewich DM, 115

Krause PJ, 249

Kremenopoulos G, 675

Kremser K, 270

Kreutzer D, 249

Kuipers JRG, 471

Kuis W, 424

Kydon DW, 323

L'Abbé MR, 293

Lacaille F, 162

Lachmann B, 490

Landaw EM, 66

Lang M, 772

Laptook AR, 370

Lasic DD, 1

Law AB, 495

Lee EL, 237

Lee SM, 560

Leffler CW, 366

Lehr H-A, 129

Leonard JL, 288

Leopaldi D, 465

Leroy A, 716

Levine JJ, 204

Levy B, 837

Levy E, 162

Levy M, 591

Lewinsky RM, 38

Lifschitz CH, 318

Li H-Q, 345

Lin D, 389

Lindén M, 308

Linderkamp O, 199, 538, 688

Lindfors A, 213

Lindstedt G, 680

Lindstrom DP, 495

Lister G, 397

Liu R, 345

Liu W, 139

Lo ES, 389

Long DR, 293

Lopaschuk GD, 735

Lorek A, 354

Lundberg P-A, 680

Mabriki W, 79

MacGillivray MH, 435

Mackay IR, 785

MacKendrick W, 222

Magagnin G, 329

Mahendran D, 661

Mailly F, 403

Maingonnat $C, 716$

Mäki M, 420

Mallampalli RK, 502

Mallard EC, 18

Mallee J, 416
Mandava S, 606

Mark A, 680

Markello TC, 115

Marquez A, 670

Marret S, 716

Martin NE, 360

Marttinen A, 420

Mast F, 44

Matsell DG, 84

Matsuda I, 258

McCawley LJ, 265

McKay CB, 538

McMicken HW, 360

Means LJ, 596

Medina JM, 709

Megyeri P, 182

Meiselman HJ, 538, 688

Melegh B, 460

Menard J, 716

Mendelsohn NJ, 694

Mendoza S, 670

Menke J, 173

Mennella JA, 805

Mevorach RA, 755

Michel E, 173

Milanesi O, 329

Milla PJ, 666

Minoo P, 729

Mirlesse V, 439

Molina RD, 654

Morrow III G, 551

Mounts KO, 596

Mulder PGH, 44, 73

Müller EE, 606

Murphey DK, 208

Nair MPN, 192

Naito $M, 258$

Nakamura R, 258

Nakamura Y, 499

Nave F, 217

Nelin LD, 349

Nguyen A, 56

Obladen M, 490

Oeseburg B, 15

Oetomo SB, 154

Oggiano N, 725

Ohno K, 105

Ohtahara S, 385

Ohta T, 258

Okken A, 154, 443

Olievier CN, 485

Oliver MH, 62

Olsson T, 495

Omiya S, 499

Oppermann M, 586

Orii T, 499

$\emptyset$ yasaeter S, 767

Ozawa T, 105

Padmanabhan V, 829

Page WV, 124

Palmer KS, 15

Pang S, 698

Pan J, 345

Pap M, 460

Paradis K, 162

Parker RA, 495, 813, 821

Parker RM, 555

Parks DA, 303

Pauly TH, 32

Peebles DM, 354

Pellegrino PA, 329

Penn R, 729

Perry VL, 187

Pesonen K, 297

Pickoff AS, 139

Plötz FB, 154
Plunkett JM, 56

Poets CF, 144

Poncelet M, 439

Porter CC, 124

Pourcyrous M, 366

Price LT, 577

Puhl H, 129

Purens J, 680

Qin S-W, 345

Quattrin T, 435

Querfeld U, 772

Qureshi IA, 777

Rabe H, 173

Radi R, 303

Raghavendra VL, 777

Raivio KO, 572

Rajaraman K, 243

Ramage D, 323

Ramji S, 809

Rebouche CJ, 460

Reddy GS, 98

Reeds PJ, 318

Reijngoud D-J, 443

Reiter EO, 829

Reynolds EOR, 354

Rhine WD, 6

Rijkers GT, 424

Ritchie JWK, 38

Riva E, 465

Rivera A, 303

Roberts RL, 544

Robillard JE, 124

Rocchiccioli F, 270

Rogers J, 416

Rognum TO, 767

Roland MO, 270

Rolfe P, 15

Roman C, 47

Rooth G, 809

Rootwelt T, 767, 809

Ross-Ascuitto NT, 323

Rowley MJ, 785

Rubin LP, 98

Rudas G, 742

Rudolph AM, 47, 51

Saeed D, 6

Saenger P, 300

Samuels MP, 144

Sanada S, 385

Sandberg K, 495

Sands M, 837

Sano T, 105

Saugstad OD, 767, 809

Schaller P, 601

Schärer K, 772

Scharpé SS, 762

Schechtman VL, 618

Schendel P, 56

Schipper IB, 178

Schmitt RM, 173

Schnackenberg U, 666

Schönli MH, 478

Schor DSM, 277

Schulze A, 601

Schwartz SA, 192

Scott ML, 66

Sedin G, 601

Segal S, 416

Segerer H, 490

Seidman E, 162

Semama DS, 120

Seri I, 742

Seyal M, 6

Shaio M-F, 720

Shapiro SM, 629

Shen C-K, 345

Sherry DD, 204

Shi Y, 345

Shine BJ, 435

Shute JK, 159

Sibley CP, 661

Siegel DM, 555

Siimes MA, 297

Silberberg AR, 495

Silverstein FS, 192

Silvestri JM, 389

Singh I, 270

Sizonenko PC, 829

Skansén-Saphir U, 213

Sladek M, 813, 82 I

Slover N, 249

Sly WS, 837

Smith CV, 360

Smith I, 10

Smith JB, 253

Solhaug MJ, 750

Sommer CW, 478

Sonesson S-E, 796

Sosa G, 6

Soubasi V, 675

Southall DP, 144

Speer CP, 182, 586

Spencer SA, 15

Sportsman C, 435

Stanley CA, 89, 265

Stebbens VA, 144

Steendijk P, 178

Sterett R, 370

Stevenson DK, 1

Stiehm ER, 66, 544

Stolfi A, 139

Stoltenberg L, 767

Stone $\mathrm{S}, 288$

Strauss AW, 694

Strickland DK, 204

Struijk PC, 44

Stuart-Smith K, 591

Sundell HW, 495, 813, 821

Surtees RAH, 10

Svanberg B, 680

Svensson L-B, 308

Swenne I, 791

Swolin B, 680

Szwarc RS, 38

Tabernero A, 709

Tabsh KMA, 253

Tahery MM, 312

Takahashi Y, 499

Takaki K, 258

Takei Y, 354

Talmud P, 403

Tanaka K, 111

Tanaka M, 105

Tang Y-W, 167

Tanikaze S, 229

Tan S, 303

Tan WKM, 18 
Traystman RJ, 530

Treem WR, 89

Truog WE, 341

Tsakiris D, 675

Tsantali C, 675

Tulassay T, 742

Tuomi T, 785

Van Acker KJ, 762

van Asselt WA, 443

van Bel F, 47, 51, 178

Van den Berghe G, 642

Van den Brande JL, 73

van der Bie W, 44

van der Bleek J, 154

van der Zee R, 424

van de Ven $C, 56,560$

van Doormaal JJ, 443

van Eden W, 424

van Gelder W, 490
Vannucci RC, 525

van Overbeek FM, 154

Van Reempts JLH, 379

van Woerkens LJPM, 490

Vázquez DM, 646

Veldhuis JD, 642

Vencato F, 329

Verkerk RM, 762

Veronikis I, 288

Vicario C, 709

Viinikka L, 297

Vilner LM, 98

Virassamy A, 624

Vitale G, 606

Vles JSH, 379

Vogler C, 837

Voorhorst-Ogink MM, 424

Vouros P, 98

Vreman HJ, I

Wagner Jr WW, 596
Walker WA, 701

Wallace MR, 750

Wang J, 698

Wang J-H, 345

Warner JO, 159

Weese-Mayer DE, 389

Wennberg RP, 6

Whelan AJ, 694

Wickramasinghe YABD, 15

Wildevuur CRH, 154

Williams CE, 18

Willi SM, 89

Wilson LE, 591

Wladimiroff JW, 44

Wolsink JG, 485

Wu KG, 544

Wu N-C, 720

Wyatt RJ, 84

Xi Y, 512
Xia Y, 634

Xie Z-W, 28

Yager J, 525

Yamada K, 606

Yamaguchi S, 111

Yamasaki S, 139

Yang KD, 720

Yang X, 698

Yeh DY, 66

Yeung B, 98

Yolken RH, 701

Young BK, 312

Young RSK, 187

Zacchello F, 329

Zegers BJM, 424

Zhang-Shanbhag L, 565

Zijlstra WG, 471

Zilow EP, 199

Zilow G, 199

Zimmet PZ, 785 